\title{
Acute Intermittent Porphyria: Characterization of a Novel Mutation in the Structural Gene for Porphobilinogen Deaminase

\author{
Demonstration of Noncatalytic Enzyme Intermediates Stabilized by Bound Substrate
}

Robert J. Desnick, Ludmila T. Ostasiewicz, Peter A. Tishler, and Pertti Mustajoki

Division of Medical Genetics, Mount Sinai School of Medicine, New York 10029; Veterans Administration Medical Center, BrocktonWest Roxbury, Massachusetts 02401 and Harvard Medical School, Boston, Massachusetts 02115; and Third Department of Medicine, University of Helsinki, 00290 Helsinki, Finland

\section{Abstract}

To investigate the molecular pathology in acute intermittent porphyria (AIP), the nature of the defective porphobilinogen (PBG)-deaminase was determined in erythrocyte lysates from 165 AIP heterozygotes from 92 unrelated families representing 20 different ethnic or demographic groups. Immunologic and physicokinetic studies revealed the occurrence of four classes of PBG-deaminase mutations. In the majority of families studied, the amount of immunoreactive enzyme protein corresponded to the amount of enzymatic activity, indicating the absence of cross-reacting immunologic material (CRIM) produced by the mutant allele. In 78 of these CRIM-negative families (designated type 1), the affected heterozygotes had half-normal PBG-deaminase activity. In three families (designated CRIM-negative type 2), symptomatic patients had increased urinary excretion of $\delta$-aminolevulinic acid and PBG, and normal levels of erythrocyte PBG-deaminase activity. In contrast, noncatalytic, immunoreactive protein was expressed in heterozygotes from 11 families, about one-eighth of those studied, consistent with mutations in the structural gene for PBG-deaminase. Two types of CRIM-positive mutations were identified: the type 1 mutation had a CRIM/activity ratio of $\sim 1.7$ and a crossed-immunoelectrophoretic profile in which all the enzyme intermediates were increased, with the $B$ or monopyrrole-enzyme intermediate predominant $(B>A \gg C \simeq D$ $>E)$. The mutation altered both the kinetic and stability properties of the noncatalytic immunoreactive enzyme protein. The second CRIM-positive mutation, type 2, had markedly increased levels of noncatalytic immunoreactive protein (CRIM/ activity ratio $\sim 5.7$ ). Crossed-immunoelectrophoresis revealed markedly increased amounts of the substrate-bound intermediates, B, C, D, and E (B $>C>D>E \gg A)$. The accumulation of these noncatalytic enzyme intermediates presumably resulted from the enhanced binding and/or defective release of substrate molecules. The conformation of these enzyme-substrate intermediates apparently rendered the complexes more resistant to intraerythrocyte proteolysis. These findings provide evidence for the presence of different allelic mutations in the structural gene for PBG-deaminase and document molecular genetic heterogeneity in AIP.

Received for publication 13 December 1984 and in revised form 18 April 1985.

J. Clin. Invest.

(c) The American Society for Clinical Investigation, Inc.

$0021-9738 / 85 / 08 / 0865 / 10 \quad \$ 1.00$

Volume 76, August 1985, 865-874
Introduction

Acute intermittent porphyria (AIP) ${ }^{1}$ is a dominantly inherited inborn error of heme biosynthesis that results from the halfnormal activity of porphobilinogen deaminase (PBG-deaminase; EC 4.3.1.8) (1-4). Clinical expression of the disease is highly variable, determined in part by environmental, metabolic, and hormonal factors that induce hepatic $\delta$-aminolevulinic acid synthase activity and the subsequent increased production of heme precursors (5-7). Although the disease is clinically latent in many heterozygous individuals, those who are symptomatic usually have elevated levels of urinary PBG and its precursor, $\delta$-aminolevulinic acid (ALA), particularly during acute attacks. Most affected individuals can be diagnosed enzymatically (i.e., decreased erythrocyte PBG-deaminase activity); however, kindreds have been described recently in which symptomatic patients have normal levels of the erythrocyte PBG-deaminase activity and markedly elevated urinary PBG and ALA levels $(8,9)$.

PBG-deaminase is encoded by a structural gene located on the distal portion of the long arm of chromosome 11 (11q23 $\rightarrow$ 11qter) (10). The erythrocyte enzyme has been purified to homogeneity and its physicokinetic properties have been characterized (11). The active enzyme protein is a monomer $\left(M_{\mathrm{r}}\right.$ $\sim 42,000$ ) that catalyzes the sequential head to tail condensation of four molecules of PBG to form hydroxymethylbilane, a linear tetrapyrrole that nonenzymatically cyclizes to uroporphyrinogen I. Hydroxymethylbilane is synthesized by a reaction mechanism involving the stepwise formation of stable enzymesubstrate intermediates (11-13). Five enzyme intermediates, A-E, have been identified by chromatographic and electrophoretic techniques, the $A$ form being the free enzyme and the $B$, $\mathrm{C}$, D, and $\mathrm{E}$ forms representing the mono-, di-, tri-, and tetrapyrrole substrate-enzyme intermediates, respectively $(11,14)$. The overall $K_{\mathrm{m}}$ for the reaction was $6 \mu \mathrm{M}$ and kinetic studies indicated that the $\mathrm{C}$ intermediate, the dipyrrole-enzyme complex, was either the most stable intermediate or the ratelimiting step in the conversion of the monopyrrole to the tetrapyrrole $(11,14)$.

In a previous communication, we reported the first immunologic characterization of the enzymatic defect in unrelated heterozygotes with AIP (14). Monospecific anti-human PBGdeaminase antibodies, which uniformly recognized each of the enzyme intermediates, were used to quantitate and characterize the amount of PBG-deaminase cross-reacting immunologic

1. Abbreviations used in this paper: AIP, acute intermittent porphyria; ALA, $\delta$-aminolevulinic acid; CRIM, cross-reacting immunologic material; DTT, dithiothreitol; PBG, porphobilinogen. 
material (CRIM) in erythrocyte lysates from 32 heterozygous individuals of 22 unrelated AIP families. Two classes of mutations were identified. In 21 of the AIP families, the amount of enzyme protein was directly proportional to the amount of enzymatic activity (i.e., the CRIM/activity ratio was $\cong 1.0$ ); these mutations were classified CRIM-negative. In seven affected members from a family of Basque ancestry, the PBG-deaminase mutation was CRIM-positive; the CRIM/ activity ratio of $\sim 1.65$ indicated the presence of immunoreactive, noncatalytic enzyme produced by the mutant allele. Although only 1 of the 22 AIP families had a CRIM-positive mutation, this finding provided evidence for heterogeneity of the genetic defect in AIP.

In this communication, we report the immunologic and physicokinetic properties of erythrocyte PBG-deaminase in 165 AIP heterozygotes from 92 unrelated families representing 20 different ethnic or demographic backgrounds. Four classes of mutations were identified: two were CRIM-negative and two were CRIM-positive. The CRIM-negative mutations were divided into two types based on the level of erythrocyte PBGdeaminase activity. The majority of CRIM-negative families, designated type 1 , had half-normal activity, whereas several CRIM-negative families, including a large Finnish kindred (8), had normal erythrocyte activity and were designated type 2 . Notably, 11 AIP families, or about one-eighth of those studied, had CRIM-positive mutations. One type was similar to that previously described in the Basque kindred (designated CRIMpositive type 1). The second type, designated CRIM-positive type 2, was unusual in that it had markedly more CRIM than expected for a single mutant allele. Immunologic studies demonstrated that the increased amount of CRIM in the type 2 heterozygotes resulted from stabilization of the mutant protein by bound substrate.

\section{Methods}

\section{Materials}

PBG was synthesized as described previously (15). Uroporphyrin standards were purchased from Porphyrin Products, Logan, UT. Diaminobenzidine tetrahydrochloride, ALA (hydrochloride), DL-dithioerythritol, DL-dithiothreitol (DTT), bovine serum albumin (BSA), and human serum albumin were obtained from Sigma Chemical Co., St. Louis, MO. High-purity acetonitrile was purchased from Burdick \& Jackson Laboratories, Muskegon, MI. Fluorescamine was obtained from Pierce Chemical Co., Rockford, IL. Goat anti-rabbit serum and peroxidase-conjugated sheep anti-rabbit IgG were obtained from Cappel Laboratories, Cochranville, PA. SeaKem agarose was purchased from Marine Colloids Div., FMC Corp., Rockland, ME. High-resolution Tris-barbital buffer was obtained from Gelman Instrument Company, Ann Arbor, MI. AgaroseIEF, Pharmalyte ampholines (pH 5-8), and Gelbond film were purchased from Pharmacia Fine Chemicals, Piscataway, NJ. Bovine pancreas trypsin was from Worthington Biochemical Corp., Freehold, NJ, and pronase B grade (Lot 200191) was from Calbiochem-Behring Corp., San Diego, CA. Stractan was obtained from the St. Regis Paper Co., Tacoma, WA. All other materials and chemicals were of the highest quality available.

\section{Human subjects and preparation of erythrocyte lysates}

Subjects included heterozygotes with AIP from unrelated families representing a variety of different ethnic backgrounds or countries of origin. The clinical diagnosis of AIP in each subject was documented by demonstration of half-normal PBG-deaminase activity in erythrocyte lysates and/or increased urinary excretion of PBG and ALA.

Heparinized blood was collected from each AIP heterozygote and
35 age- and sex-matched normal individuals, with informed consent. After centrifugation at $2,500 \mathrm{~g}$, the erythrocytes were removed, washed twice with $0.9 \% \mathrm{NaCl}$, and then used immediately or stored at $-20^{\circ} \mathrm{C}$. For specific experiments, human erythrocytes were separated into age fractions on discontinuous Stractan gradients according to the method of Corash et al. (16). Erythrocytes were lysed by the addition of 3 vol of $1.0 \mathrm{mM}$ sodium phosphate buffer, $\mathrm{pH} 8.1$, containing $1 \mathrm{mM}$ DTT, $1 \mathrm{mM} \mathrm{MgCl}$, and $0.05 \%$ Triton X-100 (lysis buffer). The lysates were centrifuged at $30,000 \mathrm{~g}$ for $20 \mathrm{~min}$ and the supernatants were removed and used for assays of protein and PBG-deaminase activity. Samples were diluted and then reassayed to insure that all lysates contained equal enzymatic activities prior to kinetic, heat denaturation, rocket immunoelectrophoretic and immunotitration studies. For protease digestion experiments, the protein concentrations of the lysates were equalized by dilution with lysis buffer.

\section{Assays}

$P B G$-deaminase assay. Enzymatic activity was determined by the quantitation of uroporphyrin fluorescence as previously described (15). One unit of enzymatic activity equaled that amount of enzyme that formed $1 \mathrm{nmol}$ of uroporphyrin per $\mathrm{h}$ at $37^{\circ} \mathrm{C}$.

Protein assay. Protein concentrations were determined by the fluorescamine procedure as previously described (17).

\section{Kinetic, heat denaturation, and protease stability studies}

For kinetic studies, $50 \mu \mathrm{l}$ of erythrocyte lysate was incubated in the standard assay with the final substrate concentration ranging from 0 to $50 \mu \mathrm{M}$. The $K_{\mathrm{m}}$ values were determined from Lineweaver-Burk plots.

To determine the effect of heat denaturation on erythrocyte PBGdeaminase activity, $500 \mu \mathrm{l}$ aliquots of the lysates, which were equalized for activity, were placed in screw-capped vials and incubated at $65^{\circ}$ or $70^{\circ} \mathrm{C}$ for $2 \mathrm{~h}$, and then cooled to $0^{\circ} \mathrm{C}$. After centrifugation at 10,000 $g$ for $10 \mathrm{~min}$ to remove precipitated proteins, $50 \mu \mathrm{l}$ of the supernatant was removed and immediately assayed for PBG-deaminase activity.

To study the effect of trypsin and pronase on PBG-deaminase activity, erythrocyte lysates were equalized for protein concentration (to $80 \mathrm{mg} / \mathrm{ml}$ by dilution with lysis buffer). Aliquots $(40 \mu \mathrm{l})$ of each lysate were placed at $0^{\circ} \mathrm{C}$, then $10 \mu \mathrm{l}$ of lysis buffer containing 125 $\mu \mathrm{g} / \mathrm{ml}$ of pronase or $0.0625 \mu \mathrm{g} / \mathrm{ml}$ of trypsin was added to give a final protease concentration of $25.0 \mu \mathrm{g} / \mathrm{ml}$ or $0.0125 \mu \mathrm{g} / \mathrm{ml}$, respectively. The reaction mixtures were preincubated for $0-60 \mathrm{~min}$ at $37^{\circ} \mathrm{C}$, after which time $500 \mu \mathrm{l}$ of $0.1 \mathrm{M}$ Tris-HCl buffer, $\mathrm{pH} 8.1$, containing 0.1 $\mathrm{mM}$ DTT, and $200 \mu \mathrm{l}$ of $0.5 \mathrm{mM}$ PBG were added immediately and the PBG-deaminase activity determined as described above.

\section{Immunologic studies}

Characterization of anti-PBG-deaminase. Anti-human PBG-deaminase was raised in New Zealand rabbits immunized with the homogeneous A intermediate and the IgG fraction was purified as previously described (14). This antibody preparation was shown to be specific for purified human PBG-deaminase (or the human enzyme in erythrocyte lysates or murine erythroleukemia-human fibroblast somatic cell hybrids) by Ouchterlony double-immunodiffusion and competitive immunoprecipitation studies $(10,14)$. Ouchterlony immunodiffusion gels showed a single arc of identity between the homogeneous enzyme and that in human erythrocyte lysates or liver homogenate supernatants when stained for protein and activity using PBG as substrate (Desnick, R. J., et al., unpublished results).

Immunotitration of anti-human PBG-deaminase. Homogeneous PBG-deaminase intermediate A (40 ng of enzyme diluted with BSA to $50 \mu \mathrm{l}$; final protein concentration of $40 \mathrm{mg} / \mathrm{ml}$ ) and $50 \mu \mathrm{l}$ of rabbit anti-PBG-deaminase IgG (in serial dilutions) were mixed and incubated at $37^{\circ} \mathrm{C}$. After $30 \mathrm{~min}, 50 \mu \mathrm{l}$ of goat anti-rabbit IgG was added and the mixture was incubated at $37^{\circ} \mathrm{C}$ for $30 \mathrm{~min}$. To insure quantitative precipitation of the anti-PBG-deaminase antibodies, the samples were left at $4^{\circ} \mathrm{C}$ overnight. The mixtures were then centrifuged at $4,000 \mathrm{~g}$ for $15 \mathrm{~min}$, and the activity in the supernatant was determined by the 
standard assay described above. For immunotitration of the immunoreactive PBG-deaminase in erythrocyte lysates from normal and AIP heterozygotes, the lysate activities were equalized and then either 50$\mu \mathrm{l}$ or $25-\mu \mathrm{l}$ aliquots were titrated against the antibody as described above.

\section{Immunologic quantitation and characterization of $P B G$ - deaminase in erythrocyte lysates}

Rocket immunoelectrophoresis. Rocket immunoelectrophoresis of human PBG-deaminase was performed as previously described (14). An agarose solution (1\%) was prepared in $0.06 \mathrm{M}$ Tris-barbital buffer, $\mathrm{pH}$ 8.8 , and bridges containing $4 \mathrm{ml}$ of agarose solution were poured into a $5 \times 7.5-\mathrm{cm}$ plate in which a double-thickness glass microscope slide had been placed in the center. After the agarose had gelled, the slide was removed and $4.0 \mathrm{ml}$ of the agarose solution containing $15 \mu \mathrm{l}$ of rabbit anti-human PBG-deaminase IgG (11 $\mu \mathrm{g}$ protein) was poured into the center area. Wells were cut at the cathodal end of the antibodycontaining gel and 5-10- $\mu$ l samples were applied. Electrophoresis was carried out in a tank (Chemtron model 200, Milan, Italy) containing $0.06 \mathrm{M}$ Tris-barbital buffer, $\mathrm{pH} 8.8$, at a constant current of $30 \mathrm{~mA}$ for $4 \mathrm{~h}$ at room temperature. After electrophoresis, the gel was thoroughly washed in $0.9 \% \mathrm{NaCl}$ for $10-12 \mathrm{~h}$ with several changes and then the antibody containing part of the gel was overlayed with $300 \mu \mathrm{l}$ of peroxidase-conjugated goat anti-rabbit IgG which had been diluted 1: 2 with $0.9 \% \mathrm{NaCl}$. The plate was incubated at room temperature in a moist chamber to prevent drying. After another overnight wash with $0.9 \% \mathrm{NaCl}$, the gel was stained for peroxidase with $50 \mathrm{ml}$ of $0.1 \mathrm{M}$ Tris- $\mathrm{HCl}$ buffer, $\mathrm{pH} 7.6$, containing $25 \mathrm{mg}$ of diaminobenzidine tetrahydrochloride and $0.15 \mathrm{ml}$ of $3 \%$ hydrogen peroxide. After staining for 10-20 min at room temperature, the rockets were visualized by using an indirect light source, and the gel was photographed and stored in $0.9 \% \mathrm{NaCl}$ at $4^{\circ} \mathrm{C}$.

Isoelectric focusing and crossed-immunoelectrophoresis. Isoelectric focusing of the PBG-deaminase intermediates was performed on horizontal agarose slab gels using the Pharmacia system according to the manufacturer's instructions. Gels were prepared by heating a mixture of $0.3 \mathrm{~g}$ of AgaroseIEF $3.6 \mathrm{~g}$ of sorbitol, and $27 \mathrm{ml}$ of distilled water in a boiling water bath. After cooling to $75^{\circ} \mathrm{C}, 1.9 \mathrm{ml}$ of $\mathrm{pH} 5-8$ Pharmalyte ampholines were added. The mixture was then poured into a horizontal casting frame $(11.4 \times 22.5 \mathrm{~cm}$; with Gelbond film backing), which had been preheated to about $60^{\circ} \mathrm{C}$ using a portable hairdryer. Gels were allowed to harden at least $1 \mathrm{~h}$ at $4^{\circ} \mathrm{C}$ or stored overnight at $4^{\circ} \mathrm{C}$ in a moist chamber. Prior to isoelectric focusing, erythrocyte lysates were heated at $60^{\circ} \mathrm{C}$ for $1 \mathrm{~h}$ and centrifuged at $10,000 \mathrm{~g}$ for $20 \mathrm{~min}$, and then aliquots of the supernatants $(50-100$ $\mu$ ) were pipetted onto Whatman No. 17 paper strips (Whatman Laboratory Products, Inc., Clifton, NJ) and placed $1 \mathrm{~cm}$ from the cathode. The cathode contained $1.0 \mathrm{M} \mathrm{NaOH}$ and the anode $0.05 \mathrm{M}$ $\mathrm{H}_{2} \mathrm{SO}_{4}$. Focusing was carried out for $2.5 \mathrm{~h}$ at $10 \mathrm{~W}$ (constant power) at $4^{\circ} \mathrm{C}$. The filter paper strips were removed after the hemoglobin had migrated about $1 \mathrm{~cm}$ from the origin. After focusing, the $\mathrm{pH}$ gradient was determined by removing a $0.5-\mathrm{cm}$ gel strip, cutting it into $1-\mathrm{cm}$ pieces which were soaked in distilled $\mathrm{H}_{2} \mathrm{O}$, and then the $\mathrm{pH}$ values of the leached ampholytes were measured. For visualization of activity bands, the gel was overlaid with Whatman No. 3MM filter paper saturated with $0.5 \mathrm{mM} \mathrm{PBG}$, covered with polyvinylchloride film, and incubated at $37^{\circ} \mathrm{C}$ for $90 \mathrm{~min}$ in the dark. The overlay was removed and the gel was exposed to ultraviolet light for $10 \mathrm{~min}$ to oxidize the uroporphyrinogen to uroporphyrin, and the red fluorescent bands of PBG-deaminase activity were rapidly photographed under ultraviolet light with Polaroid Type 58 film (Polaroid Corp., Cambridge, MA) using a Wratten No. 4 filter.

Crossed-immunoelectrophoresis of the separated PBG-deaminase intermediates was performed by placing an unstained lane from the focusing gel at the cathodal end of a rocket immunoelectrophoresis plate. The remainder of the plate was filled with $8 \mathrm{ml}$ of $1 \%$ agarose containing $40 \mu \mathrm{l}$ of anti-PBG-deaminase IgG and rocket immunoelectrophoresis was carried out as described above. Crossed-immunoelec- trophoretic gels were stained for protein with Coomassie Brilliant Blue R-250 as previously described (14).

\section{Results}

Immunologic identification of PBG-deaminase mutant classes. The PBG-deaminase CRIM status was determined in 165 AIP heterozygotes from 92 unrelated families representing at least 20 different ethnic or demographic backgrounds (Table I). For these studies, equal amounts of erythrocyte PBG-deaminase activity from each AIP heterozygote and normal individuals were subjected to rocket immunoelectrophoresis. AIP heterozygotes whose rocket peak heights were essentially identical to

Table I. Immunologic Characterization of the Defective PBGDeaminase in AIP Heterozygotes from Unrelated Families

\begin{tabular}{|c|c|c|c|c|c|c|}
\hline \multirow[b]{3}{*}{ Family ancestry* } & \multirow{3}{*}{$\begin{array}{l}\text { Number } \\
\text { of } \\
\text { families }\end{array}$} & \multirow{3}{*}{$\begin{array}{l}\text { Number } \\
\text { of } \\
\text { patients }\end{array}$} & \multicolumn{4}{|c|}{ PBG-deaminase mutant class } \\
\hline & & & \multicolumn{2}{|c|}{ CRIM-positive } & \multicolumn{2}{|c|}{ CRIM-negative } \\
\hline & & & Type 1 & Type 2 & Type 1 & Type 2 \\
\hline Basque & 1 & 7 & $x$ & & & \\
\hline Finnish & 4 & 11 & $x$ & & & \\
\hline Finnish & 2 & 4 & & $x$ & & \\
\hline Lapplander & 1 & 9 & & $x$ & & \\
\hline German & 1 & 2 & & $x$ & & \\
\hline Irish/Norwegian & 1 & 3 & & $x$ & & \\
\hline Scottish & 1 & 2 & & $x$ & & \\
\hline American Black & 6 & 7 & & & $x$ & \\
\hline Dutch & 1 & 1 & & & $x$ & \\
\hline English & 2 & 2 & & & $x$ & \\
\hline English/French & 1 & 1 & & & $x$ & \\
\hline English/German & 2 & 3 & & & $x$ & \\
\hline English/Polish & 1 & 1 & & & $x$ & \\
\hline Finnish & 23 & 38 & & & $x$ & \\
\hline Japanese & 3 & 10 & & & $x$ & \\
\hline Lapplander & 1 & 4 & & & $\times$ & \\
\hline German & 7 & 7 & & & $x$ & \\
\hline German/Dutch & 1 & 1 & & & $x$ & \\
\hline Hungarian & 1 & 1 & & & $x$ & \\
\hline Irish & 3 & 3 & & & $x$ & \\
\hline Irish/English & 1 & 1 & & & $x$ & \\
\hline Irish/Scottish & 1 & 2 & & & $\times$ & \\
\hline Italian & 5 & 6 & & & $x$ & \\
\hline Italian/French & 1 & 2 & & & $x$ & \\
\hline Norwegian & 1 & 1 & & & $x$ & \\
\hline Polish & 3 & 4 & & & $x$ & \\
\hline Spanish & 8 & 14 & & & $x$ & \\
\hline Swedish & 5 & 8 & & & $x$ & \\
\hline Swiss & 1 & 1 & & & $x$ & \\
\hline American Black & 1 & 2 & & & & $x$ \\
\hline Finnish $\ddagger$ & 1 & 5 & & & & $x$ \\
\hline Norwegian/Finnish $\ddagger$ & 1 & 2 & & & & $x$ \\
\hline Total & 92 & 165 & 5 & 6 & 78 & 3 \\
\hline
\end{tabular}

* Both parents were from the same ethnic group or country unless otherwise indicated.

$\ddagger$ AIP heterozygotes with normal erythrocyte activity. 
those of normal individuals were designated CRIM-negative (CRIM/activity ratio $\cong 1.0)$. In contrast, AIP heterozygotes whose rocket peak heights were greater than those of normal individuals were designated CRIM-positive (CRIM/activity ratio $>1.0$ ), i.e., the increased CRIM represented noncatalytic, immunoreactive PBG-deaminase expressed by the mutant allele. As shown in Fig. 1, four major PBG-deaminase mutant classes were readily identified: (a) CRIM-negative type 1 heterozygotes with half-normal PBG-deaminase activity, $(b)$ CRIM-negative type 2 heterozygotes with normal erythrocyte activity, (c) CRIM-positive type 1 heterozygotes whose peak heights were slightly greater than those of normal individuals, and $(d)$ CRIM-positive type 2 heterozygotes whose peak heights formed "railroad tracks," indicating the presence of markedly more noncatalytic PBG-deaminase than that observed in the CRIM-positive type 1 heterozygotes. Review of the medical histories did not reveal notable differences in clinical disease expression in affected individuals among the four mutant classes.

Quantitation of PBG-deaminase CRIM. The amount of immunoreactive PBG-deaminase protein in each AIP heterozygote was determined by immunotitration. When equal amounts of erythrocyte enzyme activity from CRIM-negative type 1 and 2 heterozygotes and normal individuals were immunotitrated, the same antibody dilution (1:200) was required to precipitate $50 \%$ of the activity from each of these erythrocyte supernatants and their immunotitration profiles were essentially identical (Fig. 2). The identity of their immunotitration curves confirmed the rocket immunoelectrophoretic studies that indicated the absence of noncatalytic enzyme protein in either type of CRIM-negative heterozygote. In that the amount of CRIM detected by either technique corresponded to the amount of erythrocyte activity used, the CRIM/activity ratios for the CRIM-negative type 1 and 2 heterozygotes and normal individuals were 1.0.

In contrast, immunotitration of identical amounts of activity from the CRIM-positive type 1 and 2 heterozygotes required antibody dilutions of $1: 116$ and 1:35 to precipitate $50 \%$ of the supernatant activities, respectively (Fig. 2). The antibody dilutions required to precipitate $50 \%$ of the activity from 1:1

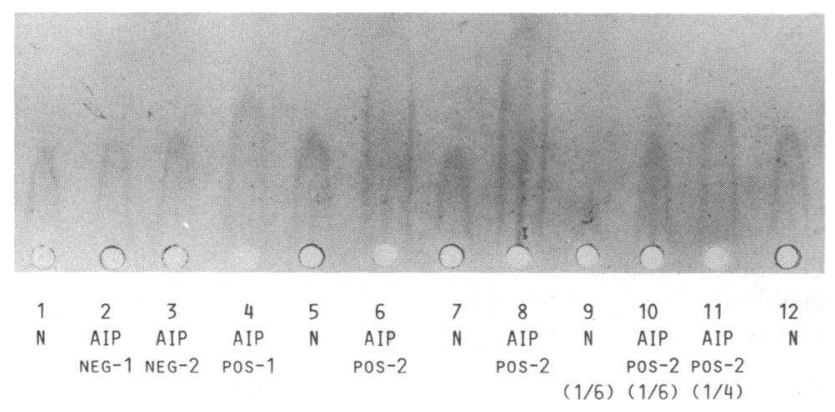

Figure 1. Rocket immunoelectrophoresis of PBG-deaminase in erythrocyte lysates from unrelated AIP heterozygotes representing each of the four mutant classes. Equal enzymatic activities were applied to the gel, except in wells 9-11 in which the activity was diluted as indicated. Normal individuals, wells 1, 5, 7, 9, and 12; CRIMnegative type 1 heterozygote (with half-normal erythrocyte activity), well 2; CRIM-negative type 2 heterozygote (with normal erythrocyte activity), well 3; CRIM-positive type 1 heterozygote, well 4; CRIMpositive type 2 heterozygote, wells $6,8,10$ and 11 .

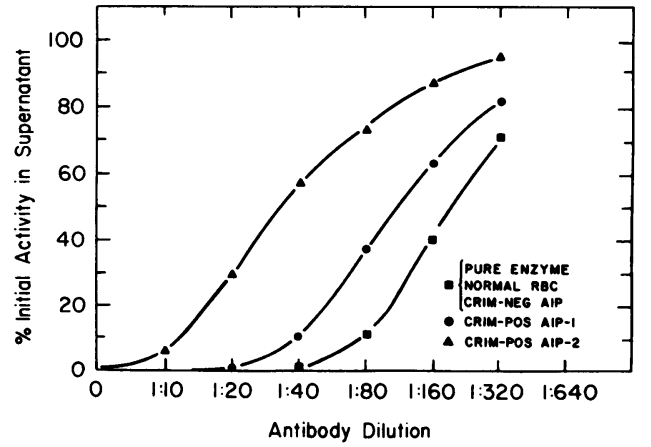

Figure 2. Immunotitration of PBG-deaminase with rabbit anti-human PBG-deaminase IgG. Immunotitration curves are shown for 25 $\mu \mathrm{l}$ aliquots (equalized for enzymatic activity and protein concentration with BSA) of homogeneous enzyme (a) and erythrocyte lysates of a normal individual ( $\square$ ), a CRIM-negative type 1 heterozygote $(\boldsymbol{\square})$, a CRIM-positive type 1 heterozygote (๑), and a CRIM-positive type 2 heterozygote ( $\triangle$ ). Note that the curves for $25 \mu \mathrm{l}$ of the homogeneous enzyme, normal lysate, and the CRIM-negative type 1 lysate were essentially identical; the curve for $25 \mu \mathrm{l}$ of the CRIM-negative type 2 lysate also was identical (data not shown). Antibody dilutions of $\sim 1$ : $35,1: 116$ and 1:200 were observed for 50\% immunoprecipitation of enzyme from $25 \mu$ l of the CRIM-positive type 2 lysate, the CRIMpositive type 1 lysate and the CRIM-negative type 1 (or normal) lysate, respectively.

mixtures of normal and CRIM-positive type 1 or type 2 lysates were $1: 146$ and 1:60, respectively (data not shown). Based on these results, and assuming identical antibody avidities, the amounts of immunoreactive PBG-deaminase in these representative CRIM-positive type 1 and 2 heterozygotes were $\sim$ 1.7- and 5.7-fold that in normal lysates (i.e., CRIM/activity ratios of 1.7 and 5.7). Analogously, the CRIM/activity ratios determined by immunotitration for the 18 CRIM-positive type 1 and the 20 CRIM-positive type 2 heterozygotes ranged from 1.6 to $1.8($ mean $\pm 1 S D=1.7 \pm 0.07)$ and from 4.9 to 6.0 $($ mean $\pm 1 \mathrm{SD}=5.7 \pm 0.3)$, respectively.

The amount of immunoreactive PBG-deaminase in the CRIM-positive type 2 heterozygotes also was determined by quantitative rocket immunoelectrophoresis. As shown in Fig. 3, the rocket peak heights for 7 and $10 \mathrm{mU}$ of PBG-deaminase activity in the CRIM-positive type 2 erythrocytes were essentially equal to those obtained for 40 and $56 \mathrm{mU}$ of normal lysate activity, respectively. Thus, the CRIM/activity ratio determined by rocket immunoelectrophoresis was $\sim 5.7$, consistent with the values for the CRIM-positive type 2 heterozygotes obtained by immunotitration.

Characterization of the PBG-deaminase intermediates in the AIP mutant classes. Crossed-immunoelectrophoresis of erythrocyte PBG-deaminase provided further information concerning the nature of the enzymatic defect in the four AIP mutant classes. For these studies, PBG-deaminase in erythrocyte lysates was first subjected to isoelectric focusing in agarose gels to separate the enzyme intermediates. Five major PBG-deaminase activity bands were observed in normal and CRIMpositive type 1 and 2 lysates (Fig. 4), as well as in CRIMnegative type 1 and 2 lysates (data not shown). There were no significant differences in the pI values for each activity band in lysates from the different mutant classes. After crossedimmunoelectrophoresis, a precipitin line of identity with peaks corresponding to each of the five intermediates was observed 


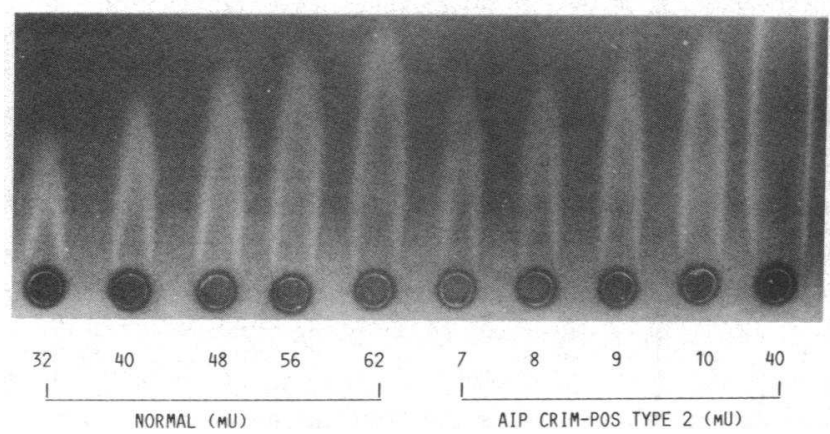

Figure 3. Estimation of the immunoreactive PBG-deaminase in a CRIM-positive type 2 heterozygote. From left to right: wells 1-5 contained equal increments of normal erythrocyte PBG-deaminase activity (32-62 $\mathrm{mU})$ and wells 6-9 contained equal increments of lysate activity (7-10 mU) from the CRIM-positive type 2 heterozygote. Note that the peak heights for 40 and $56 \mathrm{mU}$ of normal lysate activity were about equal to those obtained for 7 and $10 \mathrm{mU}$ of the CRIM-positive type 2 lysate. Thus, the ratio of immunoreactive PBG-deaminase to activity (CRIM/activity ratio) for the CRIM-positive type 2 heterozygote was $\sim 5.7$. See text for details.

in lysates from normal individuals and AIP heterozygotes from each mutant class. The typical profiles obtained for erythrocyte PBG-deaminase from normal individuals and all CRIM-negative type 1 and 2 heterozygotes are shown in Fig. 4. In normal lysates, the $A$ intermediate had the highest peak height, with $\mathrm{B}, \mathrm{C}, \mathrm{D}$, and $\mathrm{E}$ intermediates having sequentially decreasing peak heights (upper gel). In contrast, lysates from CRIMpositive type 1 heterozygotes (middle gel) had increased amounts of all five intermediates, the immunoreactive $B$ intermediate being most elevated (14). Lysates from the CRIMpositive type 2 heterozygotes (lower gel) had a distinctly different profile: the A peak appeared decreased whereas the $\mathrm{B}, \mathrm{C}, \mathrm{D}$, and $\mathrm{E}$ peaks were markedly increased. For comparison, the amount of type 2 activity applied to this gel was one-fifth of that used for crossed-immunoelectrophoresis of the normal or CRIM-positive type 1 enzyme.

To further characterize the amount of immunoreactive PBG-deaminase intermediates in CRIM-positive type 2 heterozygotes, the erythrocyte enzyme intermediates from CRIMpositive type 2 and normal individuals were isolated by DEAEcellulose chromatography (14), and equal activities of the A, B, C, and D forms were subjected to rocket immunoelectrophoresis. As shown in Fig. 5, each of the intermediates from the CRIM-positive type 2 lysate had an increased amount of immunoreactive enzyme protein when compared with that obtained with the respective normal intermediate. Notably, the CRIM-positive B, C, and D intermediates were markedly increased whereas the $A$ form, the free enzyme, was only slightly increased.

Characterization of the immunoreactive PBG-deaminase in age-fractionated erythrocytes from the CRIM-positive type 2 heterozygotes. Erythrocytes from a CRIM-positive type 2 heterozygote and a normal individual were fractionated into age cohorts on discontinuous Stractan density gradients, according to the method of Corash et al. (16). As shown in Fig. 6, four age-cohort fractions were obtained from a CRIM-positive heterozygote and five fractions were obtained from normal erythrocytes. These fractions ranged in mean age from 30 to $\sim 90 \mathrm{~d}$. The lysates of the normal erythrocyte fractions (youngest

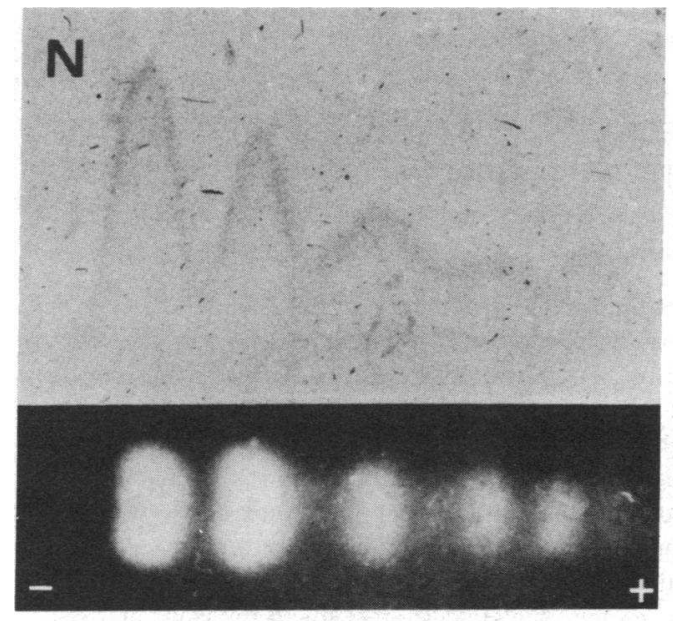

A B $\quad$ C $\quad$ D $E$

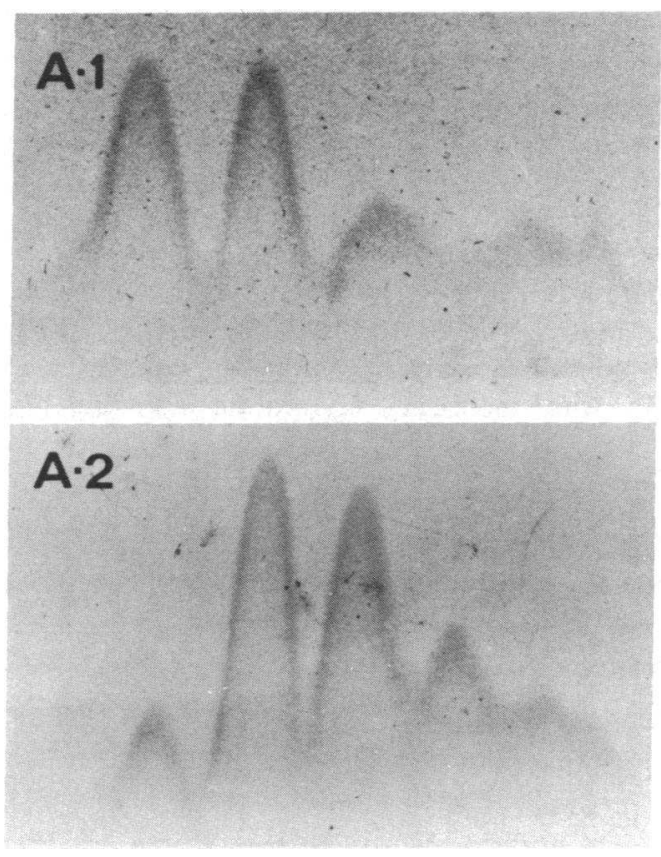

Figure 4. Isoelectric focusing and crossed-immunoelectrophoresis of PBG-deaminase in erythrocyte lysates from normal individuals $(N$; $40 \mathrm{mU})$ and CRIM-positive type $1(A-1 ; 40 \mathrm{mU})$ and type $2(A-2 ; 8$ $\mathrm{mU})$ heterozygotes. Isoelectric focusing revealed five bands when stained for activity in normal, CRIM-positive type 1 and 2, as well as CRIM-negative type 1 and 2 lysates (not shown). No significant differences in the $\mathrm{pI}$ values for each band in the different lysates were observed. After crossed-immunoelectrophoresis of the focused lysate proteins, only immunoreactive proteins corresponding to the activity bands were observed in the normal, CRIM-positive type 1 and 2 (or CRIM-negative type 1 or 2; not shown) heterozygotes. Note the distribution of immunoreactive enzyme intermediates in the CRIMpositive type 1 and 2 heterozygotes compared to those in the normal erythrocyte lysate. See text for details.

to oldest) had PBG-deaminase activities of $0.159,0.126,0.102$, 0.095 , and $0.081 \mathrm{U} / \mathrm{mg}$ protein compared to 0.103 for the unfractionated lysate. The lysate activities of the fractionated CRIM-positive type 2 heterozygote (youngest to oldest) were 


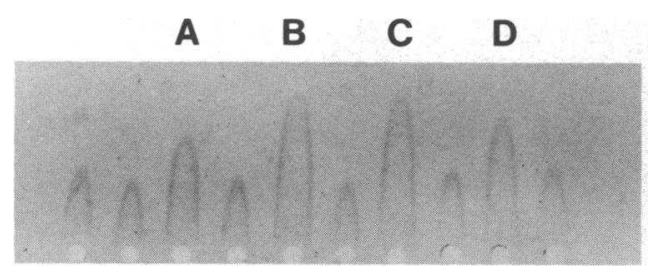

Figure 5. Rocket immunoelectrophoresis of PBG-deaminase intermediates in erythrocytes from a CRIM-positive type 2 heterozygote and a normal individual. Equal activities of each intermediate, isolated by DEAE-cellulose chromatography, were immunoelectrophoresed. From left to right: wells $1,2,4,6,8$ and 10, normal erythrocyte PBG-deaminase intermediates A, A, B, C, D, and A, respectively; wells 3, 5, 7 and 9, CRIM-positive type 2 PBG-deaminase intermediates $A, B, C$, and $D$, respectively. Note that each intermediate from the CRIM-positive type 2 heterozygote had an increased amount of immunoreactive protein, the greatest amounts in the B, C, and D or substrate-bound intermediates.

$0.136,0.077,0.054$, and $0.050 \mathrm{U} / \mathrm{mg}$ protein, whereas the activity in the unfractionated lysate was $0.068 \mathrm{U} / \mathrm{mg}$. As shown in Fig. 7 (upper gel), rocket immunoelectrophoresis of equal amounts of activity from the unfractionated normal lysate and from the age-fractionated lysates (youngest to oldest) revealed no difference in rocket peak height, indicating that the amount of activity corresponded to the amount of immunoreactive enzyme protein in each age-fraction. In contrast, rocket immunoelectrophoresis of 0.2 activity units of the unfractionated lysate, and the four age-fractionated lysates from a type 2 heterozygote, demonstrated increasing amounts of cross-reactive enzyme protein with increasing erythrocyte age (Fig. 7, lower gel).

As shown in Fig. 8, crossed-immunoelectrophoresis of a lysate from the youngest type 2 age fraction revealed a profile in which the $B$ intermediate had the highest peak height

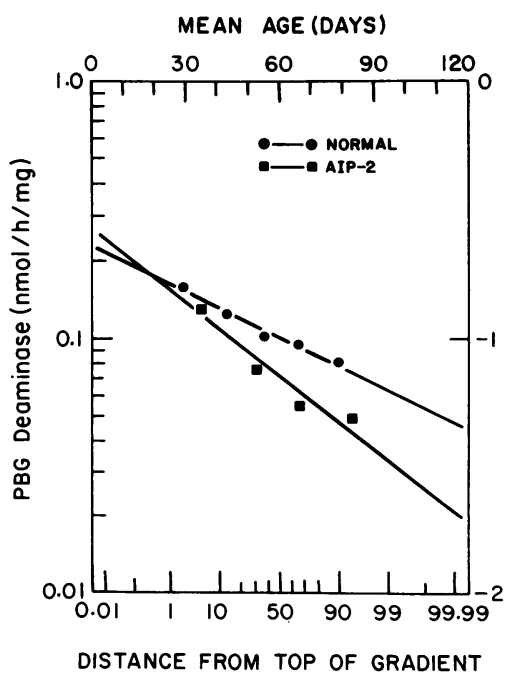

Figure 6. Age-fractionation of erythrocytes from a normal individual (•) and a CRIM-positive AIP type 2 heterozygote (口). Erythrocytes were separated in discontinuous Stractan gradients by isopycnic centrifugation (16). Five fractions with mean ages of $\sim 30,43,55,66$, and $80 \mathrm{~d}$ were separated from normal erythrocytes. Fqur fractions with mean ages of $35,52,66$, and $84 \mathrm{~d}$ were separated from a CRIM-positive type 2 heterozygote.

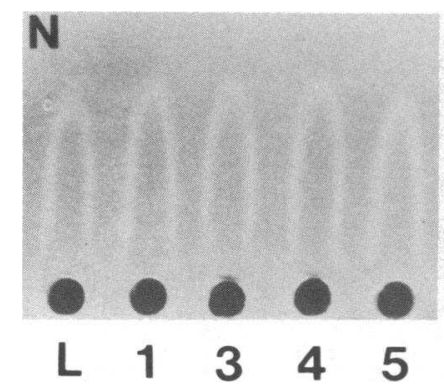

Figure 7. Rocket immunoelectrophoresis of PBG-deaminase in age-fractionated erythrocyte lysates from a normal individual $(N)$ and a CRIM-positive AIP type 2 heterozygote $(A-2)$. Equal amounts of enzymatic activity were applied in each well. No differences were observed in the rocket peak

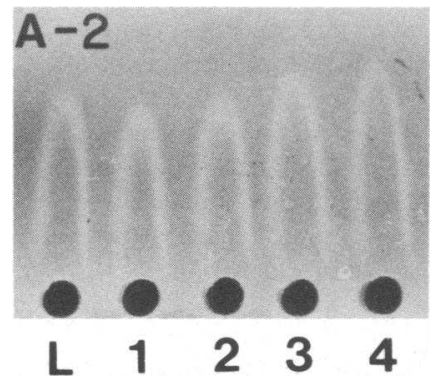
heights of immunoreactive enzyme in the normal lysate $(L)$ or normal age-fractions (wells $1-5$, youngest to oldest). In contrast, increasing amounts of immunoreactive enzyme were observed in the age-fractionated lysates (wells 1-4, youngest to oldest) from the CRIMpositive AIP type 2 heterozygote.

(middle gel), whereas crossed-immunoelectrophoresis of a lysate from the oldest type 2 age fraction (lower gel) revealed a profile in which the $\mathrm{C}$ intermediate was the highest peak. Presumably, the $\mathrm{C}$ or dipyrrole-enzyme intermediate represents
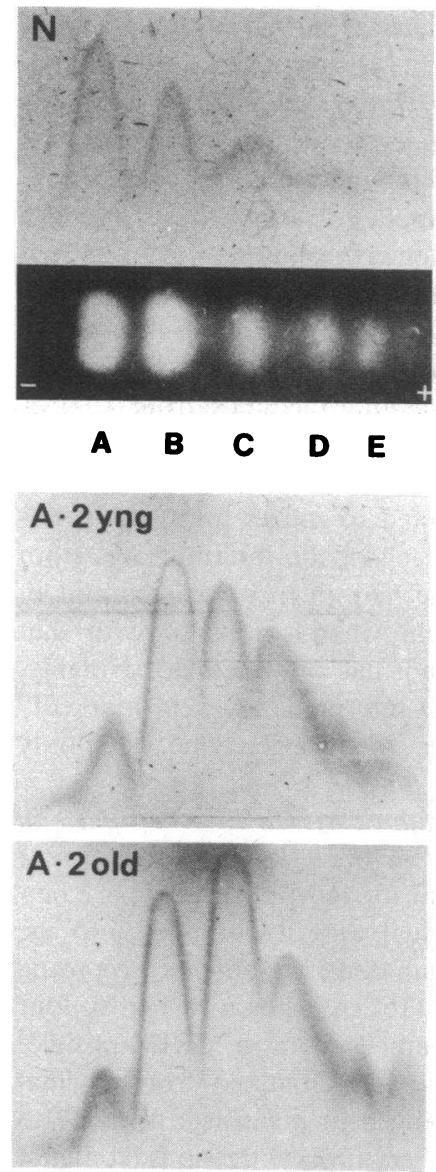

Figure 8. Crossed-immunoelectrophoresis of PBG-deaminase in normal erythrocytes $(N)$ and in age-fractionated erythrocytes ( $A$-2yng, youngest; $A$-2old, oldest) from a CRIM-positive AIP type 2 heterozygote. Note the different profiles of the intermediates in the youngest (B $>\mathrm{C}>\mathrm{D}>\mathrm{E}>\mathrm{A})$ and oldest $(\mathrm{C}>\mathrm{B}>\mathrm{D}>\mathrm{E}>\mathrm{A})$ age fractions. 
the rate-limiting step in the sequential conversion of the monopyrrole to the linear tetrapyrrole and/or the most stable enzyme-substrate complex $(11,14)$.

Kinetic, heat denaturation, and protease stability studies. The physicokinetic properties of the PBG-deaminase in erythrocyte lysates from normal individuals and each of the AIP mutant classes are summarized in Table II. The $K_{\mathrm{m}}$ values calculated from Lineweaver-Burk plots were essentially the same $(\sim 6 \mu \mathrm{M})$ from all sources. However, heat denaturation and protease digestion studies revealed differences among the AIP mutant classes. When the lysates were heat denatured at $65^{\circ}$ and $70^{\circ} \mathrm{C}$, the CRIM-positive type 2 enzyme was most stable and was slightly activated at $65^{\circ} \mathrm{C}$. The CRIM-positive type 1 and CRIM-negative type 1 activities had mean heat inactivation values that were similar to those of the normal enzyme. Interestingly, the CRIM-negative type 2 activity was less stable than the normal enzyme after heat treatment at $65^{\circ} \mathrm{C}$. When equal amounts of erythrocyte protein from each of the mutant classes were subjected to pronase or trypsin digestion, the activities of the CRIM-positive type 1, both CRIM-negative type 1 and 2 heterozygotes, and normal indi- viduals were equally digested by either protease. In contrast, the CRIM-positive type 2 activity was more stable to pronase digestion and appeared slightly more stable to trypsin degradation. Rocket immunoelectrophoresis of equal activities of each mutant class did not reveal any differences in the peak height of the enzyme before or after pronase or trypsin digestion (data not shown), suggesting that the partially degraded enzyme proteins lost activity, but fully retained their antigenic properties.

\section{Discussion}

Four major mutant classes were identified by immunologic and physicokinetic studies of PBG-deaminase in erythrocytes from unrelated AIP heterozygotes with this dominantly inherited, inborn error of metabolism. Two of these mutant classes had been defined previously by immunologic studies of heterozygotes from 22 unrelated AIP families (14). By using monospecific antibodies to quantitate immunoreactive enzyme protein, the heterozygotes from all but one of these families were found to be CRIM-negative (CRIM/activity ratio $\sim 1.0$ ),

Table II. Comparison of the PBG-Deaminase Mutant Classes

\begin{tabular}{|c|c|c|c|c|c|}
\hline \multirow[b]{3}{*}{ Property } & \multirow[b]{3}{*}{ Normal } & \multicolumn{4}{|c|}{ Acute intermittent porphyria } \\
\hline & & \multicolumn{2}{|c|}{ CRIM-negative } & \multicolumn{2}{|l|}{ CRIM-positive } \\
\hline & & Type 1 & Type 2 & Type 1 & Type 2 \\
\hline \multicolumn{6}{|c|}{ Specific activity ( $U / m g$ protein) } \\
\hline Mean & 0.063 & 0.030 & 0.060 & 0.032 & 0.034 \\
\hline $\pm 1 \mathrm{SD}$ & 0.008 & 0.004 & 0.008 & 0.003 & 0.004 \\
\hline$(n)$ & $(35)$ & (118) & (9) & $(18)$ & $(20)$ \\
\hline \multicolumn{6}{|l|}{ CRIM/activity ratio } \\
\hline Mean & 1.0 & 1.0 & 1.0 & 1.7 & 5.7 \\
\hline $\pm 1 \mathrm{SD}$ & 0.06 & 0.05 & 0.06 & 0.09 & 0.23 \\
\hline$(n)$ & $(35)$ & (118) & (9) & (18) & (20) \\
\hline Apparent $K_{\mathrm{m}}(\mu M)$ & 5.9 & 5.7 & 6.0 & 5.5 & 5.9 \\
\hline \multicolumn{6}{|l|}{ Heat stability* (\% int. act.) } \\
\hline \multicolumn{6}{|l|}{$65^{\circ} \mathrm{C}$} \\
\hline Mean $(n=6)$ & 95 & 95 & 85 & 99 & 106 \\
\hline Range & $(89-101)$ & $(92-100)$ & $(82-87)$ & $(93-106)$ & $(95-115)$ \\
\hline \multicolumn{6}{|l|}{$70^{\circ} \mathrm{C}$} \\
\hline Mean $(n=6)$ & 62 & 60 & 58 & 60 & 62 \\
\hline Range & $(52-66)$ & $(53-66)$ & $(54-61)$ & $(59-61)$ & $(60-63)$ \\
\hline \multicolumn{6}{|c|}{ Protease Stability (\% int. act.) } \\
\hline \multicolumn{6}{|c|}{ Pronase } \\
\hline Mean $(n=6)$ & 39 & 35 & 39 & 41 & 56 \\
\hline Range & $(31-48)$ & $(26-51)$ & $(23-50)$ & $(31-56)$ & $(42-67)$ \\
\hline \multicolumn{6}{|l|}{ Trypsin } \\
\hline Mean $(n=6)$ & 72 & 67 & 71 & 69 & 74 \\
\hline Range & $(63-79)$ & $(60-74)$ & $(62-79)$ & $(51-81)$ & $(63-85)$ \\
\hline Isoelectric focusing profile & Nlł & $\mathrm{Nl}$ & $\mathrm{Nl}$ & $\mathrm{Nl}$ & $\mathrm{Nl}$ \\
\hline \multicolumn{6}{|c|}{ Crossed-Immunoelectrophoresis } \\
\hline Profile & $\mathrm{Nl}$ & $\mathrm{Nl}$ & $\mathrm{Nl}$ & $\mathrm{B}>\mathrm{A} \gg \mathrm{C}>\mathrm{D}>\mathrm{E}$ & $\mathrm{B}>\mathrm{C} \gg \mathrm{D}>\mathrm{E} \gg \mathrm{A}$ \\
\hline
\end{tabular}

* Stability after $120 \mathrm{~min}$ at $65^{\circ} \mathrm{C}$ and $70^{\circ} \mathrm{C}$ expressed as percent of initial activity (\% int. act.). $\ddagger \mathrm{N} 1=\mathrm{A}>\mathrm{B} \gg \mathrm{C}>\mathrm{D}>\mathrm{E}$ profile on isoelectric focusing and crossed-immunoelectrophoresis. 
i.e., immunoreactive, noncatalytic enzyme protein encoded by the mutant allele was not detectable in erythrocyte lysates. In only one of 22 AIP families, the mutation was CRIM-positive (CRIM/activity ratio $\sim 1.7$ ), consistent with a structural gene mutation in an allele encoding PBG-deaminase. In this communication, two additional PBG-deaminase mutant classes, designated CRIM-negative type 2 and CRIM-positive type 2, were identified and characterized. Each of these mutations was novel and provided further insight into the nature of the defects underlying the PBG-deaminase deficiency in AIP.

Characterization of the CRIM-positive mutations was permitted by the recognition that PBG-deaminase catalyzed the formation of hydroxymethylbilane via five stable enzyme intermediates, designated A-E (11-13). Previously, a model was proposed (Fig. 9), which predicted the different types of mutations which might be revealed by immunologic characterization of the PBG-deaminase intermediates in CRIMpositive heterozygotes (14). This model assumes that the normal enzyme has two binding sites for PBG and one active site (18), with the normal reaction mechanism involving the stepwise formation of the mono-, di-, tri-, and tetrapyrroles by the enzyme-substrate intermediates, A-E. Mutations that render substrate-binding site 1 defective would not permit PBG binding, and would result in the accumulation of the free enzyme. A mutation in the second substrate-binding site would lead to the accumulation of both the $B$ and $A$ intermediates, presumably with more $B$ than $A$. If a mutation resulted in altered binding and/or catalysis, a $K_{\mathrm{m}}$ mutation would result and all of the enzyme-substrate forms would accumulate, perhaps the $\mathrm{B}$ or $\mathrm{C}$ form in greater amounts, depending on whether the substrate-binding or active site was more defective. If a mutation resulted in increased binding and/or defective release of the product, only the substrate-bound forms would accumulate and, presumably, the A form would not be increased.
Immunologic characterization of the CRIM-positive type 1 mutation revealed that each of the enzyme intermediates had elevated levels of cross-reactive material and the noncatalytic B enzyme-substrate intermediate was present in the greatest amount as previously described (14). These findings best fit the model for a $K_{\mathrm{m}}$ mutation with a markedly altered, but functional, active site and an altered substrate-binding site 2 (Fig. 9). In addition, the immunoreactive gene product produced by the mutant allele was somewhat unstable, because the CRIM/activity ratio was 1.7 , whereas a totally stable mutant protein would have had a CRIM/activity ratio of 2.0 in the heterozygous lysate.

In marked contrast, the CRIM-positive type 2 mutants had a CRIM/activity ratio of $\sim 5.7$ (range 4.9-6.0), an unexpected finding, and a crossed-immunoelectrophoretic profile in which the immunoreactive $\mathrm{B}, \mathrm{C}, \mathrm{D}$, and $\mathrm{E}$ enzyme-substrate intermediates were markedly elevated, while the A intermediate, the free enzyme, was only slightly increased. These findings best fit the model, which predicted increased binding or defective release of the bound substrate molecules (Fig 9). It appears that the substrate-bound intermediates are particularly resistant to degradation in the erythrocyte, as evidenced by the increasing CRIM-activity ratio in the older age-fractionated erythrocytes (Figs. 7 and 8). Thus, the type 2 structural gene mutation represents a unique type of stability defect, one in which the nonfunctional mutant protein is more stable to in vivo proteolysis than the normal, catalytically active erythrocyte enzyme. Of the numerous human protein mutations characterized to date, only two hyperstable variants have been identified, hemoglobins Hacettepe and Agenogi (19-21). In each of these mutant hemoglobins, a $\beta$-chain amino acid substitution resulted in the formation of new ionic bonds in the molecule which rendered the mutant proteins more stable to heat denaturation than the normal protein. By analogy, an amino acid substitution in the mutant PBG-deaminase mono-
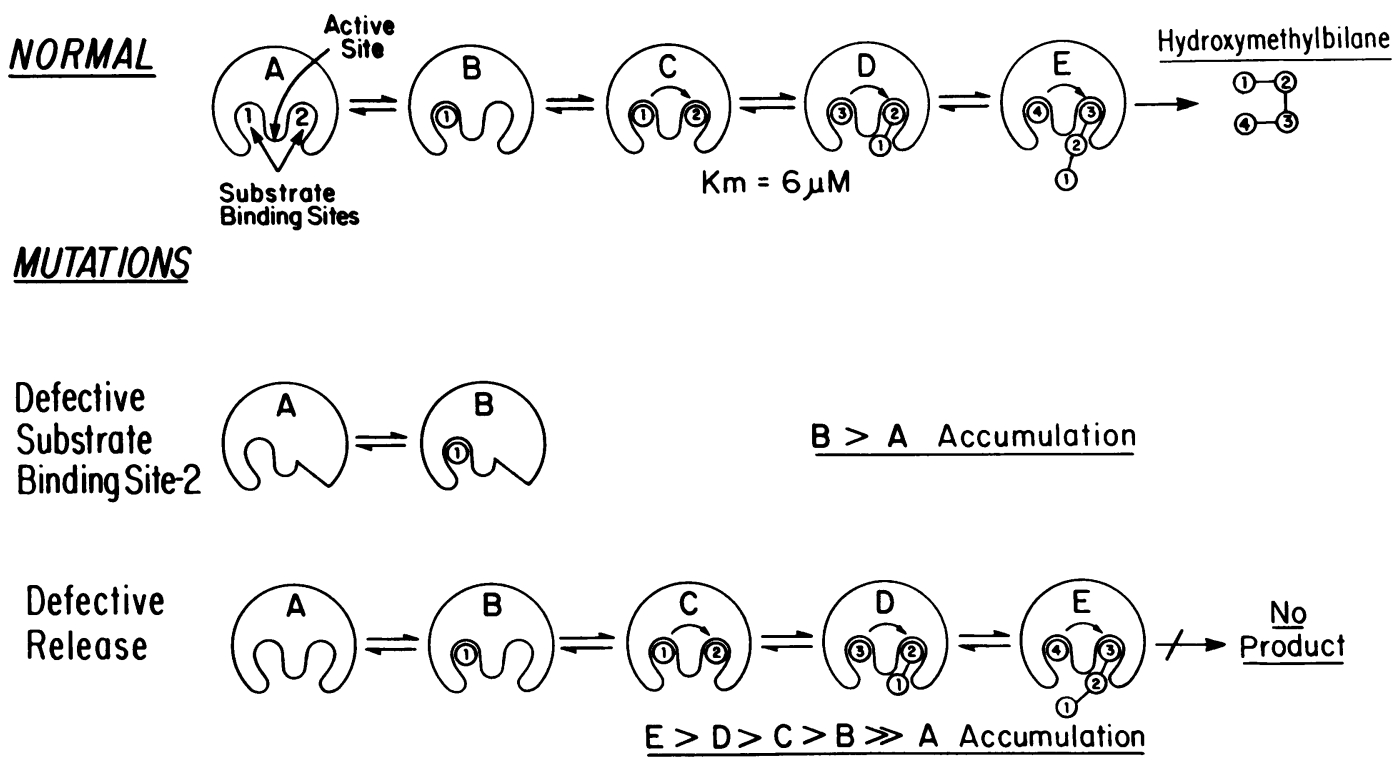

Figure 9. Possible alterations in the CRIM-positive type 1 and 2 noncatalytic enzyme proteins resulting from different mutations in the PBG-deaminase structural gene. The normal enzyme is assumed to have one active site and two substrate-binding sites; the stepwise formation of the mono-, di-, tri-, and tetrapyrrole-enzyme intermedi- ates (B, C, D, and E, respectively) is shown. Mutations which alter substrate-binding site 2 and product release as well as the expected formation and/or accumulation of the enzyme intermediates for each are depicted. See Discussion for details. 
mer could markedly alter substrate binding and/or product release rendering the mutant substrate-enzyme complexes more stable. Of particular interest was the observation that the presence of the immunoreactive, noncatalytic enzyme in these heterozygotes altered the heat and protease stabilities of the activity expressed by the normal allele. The mechanism by which the accumulated mutant enzyme stabilized the normal erythrocyte activity remains unclear. Because PBG-deaminase is an extremely hydrophobic protein, one possible explanation might be that the normal and mutant proteins aggregate, thereby permitting the hyperstable mutant protein to increase the stability of the normal enzyme.

The CRIM-negative type 2 mutation was differentiated from the CRIM-negative type 1 mutation by the fact that the level of erythrocyte PBG-deaminase activity in these individuals was within the normal range. The normal erythrocyte PBGdeaminase levels in these individuals did not result from elevated reticulocyte counts, nor were they due to the biologic range in the expression of a single normal allele (e.g., high heterozygote level), since the activity ratios of erythrocyte PBG-deaminase to $\delta$-aminolevulinate dehydratase and uroporphyrinogen III cosynthase were normal (Desnick, R. J., unpublished observations). Several hypotheses have been advanced to account for the normal erythrocyte PBG-deaminase activities in these symptomatic patients. It has been suggested that these AIP heterozygotes have half-normal hepatic PBG-deaminase activities and normal erythrocyte levels (8). This hypothesis requires the presence of two structural genes encoding hematopoietic (e.g., erythrocytic) and nonhematopoietic (e.g., hepatic) isozymes or a regulatory mechanism which controls either the tissue specific expression of a single structural gene or the differential expression of hematopoietic and nonhematopoietic isozymes. Support for this concept will await determination of the hepatic activity in these patients. However, the fact that human PBG-deaminase has been assigned to a single, narrow, chromosomal location (10) and the finding that monospecific antibodies against the purified erythrocyte enzyme form a single line of identity with partially purified hepatic and erythrocytic PBG-deaminase activities, argue against this hypothesis (Ostasiewicz, L. T., and R. J. Desnick, unpublished results). Nevertheless, it is possible that two or more structural genes are present as a gene family in the same chromosomal region, analogous to the $\beta$-globin gene complex (22), and that the antierythrocyte PBG-deaminase antibodies did not recognize unique determinants of a hepatic isozyme. Another explanation which also may depend on the occurrence of separate genes encoding hepatic and erythrocytic enzymes, is the suggestion that the defect in AIP results from a regulatory gene mutation $(7,23)$. Such a mutation could result in normal hematopoietic, but half-normal hepatic gene expression.

Finally, one could account for the rare individuals with normal erythrocyte activity by the segregation of a variant allele with high normal activity (i.e., a hypermorph). It is well known that there is a large range (about threefold) in normal erythrocytic PBG-deaminase levels and that this variation is genetically determined $(24,25)$. The occurrence of two codominant PBG-deaminase alleles was hypothesized recently to account for the distribution of the erythrocyte activities in over 200 normal individuals (26). For the two-allele model, the mean activities for the trimodal distributions were $\sim 130$, 170 , and $220 \mathrm{pmol} / 30 \mathrm{~min} \cdot \mathrm{mg}$ protein, respectively. If an individual inherited such a hypermorphic allele and an AIP mutant allele, the level of erythrocyte PBG-deaminase activity may be within the normal range (but the nonhematopoietic activities could be half-normal). Symptomatic individuals in such a family have been described (27); normal individuals with the hypermorphic allele had high normal activities of erythrocyte PBG-deaminase, whereas symptomatic heterozygous individuals, who had the hypermorphic allele and a mutant AIP allele, had erythrocyte activities in the normal range. However, characterization of the erythrocyte enzyme in six asymptomatic, apparently normal members of the large Finnish CRIM-negative type 2 family did not reveal activity levels consistent with the presence of a hypermorphic allele. Therefore, it is likely that insight into the precise nature of the defect in these CRIM-negative type 2 heterozygotes will be revealed by molecular studies of their PBG-deaminase genes.

In summary, immunologic and biochemical characterization of the enzymatic defect in a large series of unrelated AIP families identified four classes of PBG-deaminase mutations. At the molecular level, the two CRIM-positive mutations most likely result from single base substitutions in the exons of the structural gene. The CRIM-negative mutations may represent mutations which $(a)$ markedly alter enzyme stability or antigenicity, (b) result in early chain termination, or (c) alter mRNA transcription or processing. The classification of these mutations should facilitate the initial selection of appropriate candidates for future molecular genetic analyses, analogous to the characterization of the genetic defects in the human thalassemias (22).

\section{Acknowledgments}

The authors wish to thank Dr. David Bishop for helpful discussions and suggestions, Mr. Raman Reddy for expert technical assistance, Dr. Sergio Piomelli and Ms. Carol Seaman for assistance with the separation of erythrocyte age fractions, and Mrs. Linda Lugo for her expert clerical assistance. The authors give special thanks to the many AIP heterozygotes who, together with their physicians, Drs. Karl Anderson, Alcira Batlle, Paul Berk, Christopher Calder, Bertram Felsher, Mary Hand, Arvid Heiberg, Joseph Herch, Fritz Hommes, Claus Pierach, Randolf Schiffer, William Sly, and Gumpei Urata, provided the erythrocytes for these studies.

This research was supported in part by a research grant (1-535) from the March of Dimes Birth Defects Foundation and a grant (AM26824) from the National Institutes of Health. Ludmila T. Ostasiewicz is the recipient of an National Institutes of Health predoctoral fellowship (2 T32 HD-07105) in medical genetics.

\section{References}

1. Strand, L. J., B. F. Felsher, A. G. Redeker, and H. S. Marver. 1970. Enzymatic abnormality in heme biosynthesis in intermittent acute porphyria: decreased hepatic conversion of porphobilinogen to porphyrins and increased delta aminolevulinic acid synthetase activity. Proc. Natl. Acad. Sci. USA. 67:1315-1320.

2. Miyagi, K., R. Cardinal, I. Bossenmaier, and C. J. Watson. 1971. The serum porphobilinogen and hepatic porphobilinogen deaminase in normal and porphyric individuals. J. Lab. Clin. Med. 78: 683-695.

3. Strand, L. J., U. A. Meyer, B. F. Felsher, A. G. Redeker, and H. S. Marver. 1972. Decreased red cell uroporphyrinogen I synthetase activity in intermittent acute porphyria. J. Clin. Invest. 51:2430-2536.

4. Meyer, U. A., L. J. Strand, M. Doss, A. C. Rees, and H. S. Marver. 1972. Intermittent acute porphyria: demonstration of a genetic defect in porphobilinogen metabolism. N. Engl. J. Med. 286:12771282. 
5. Meyer, U., and R. Schmid. 1978. The porphyrias. In The Metabolic Basis of Inherited Disease. J. B. Stanbury, J. B. Wyngaarden, and D. S. Fredrickson, editors. Fourth edition. McGraw-Hill Book Company, New York. 1166-1220.

6. Tschudy, D. P., and J. M. Lamon. 1980. Porphyrin metabolism and the porphyrias. In Metabolic Control and Disease. P. K. Bondy and L. Rosenberg, editors. Eighth edition. W. B. Saunders Co., Philadelphia. 939-1007.

7. Kappas, A., S. Sassa, and K. E. Anderson. 1983. The porphyrias. In The Metabolic Basis of Inherited Disease. J. B. Stanbury, J. B. Wyngaarden, D. S. Fredrickson, J. L. Goldstein, and M. S. Brown, editors. Fifth edition. McGraw-Hill Book Company, New York. 13011384.

8. Mustajoki, P. 1981. Normal erythrocyte uroporphyrinogen I synthase in a kindred with acute intermittent porphyria. Ann. Intern. Med. 95:162-166.

9. Astrup, E. G. 1978. Family studies on the activity of uroporphyrinogen I synthase in diagnosis of acute intermittent porphyria. Clin. Sci. Mol. Med. 54:251-156.

10. Wang, A.-L., F. X. Arredondo-Vega, P. F. Giampietro, M. Smith, W. F. Anderson, and R. J. Desnick. 1981. Regional gene assignments for porphobilinogen deaminase and esterase- $\mathrm{A}_{4}$ on chromosome 11. Proc. Natl. Acad. Sci. USA. 78:5734-5738.

11. Anderson, P. M., and R. J. Desnick. 1980. Purification and properties of uroporphyrinogen I synthase from human erythrocytes: identification of stable enzyme-substrate intermediates. J. Biol. Chem. 255:1993-1999.

12. Battersby, A. R., C. J. R. Fookes, G. W. J. Matcham, and E. McDonald. 1980. Biosynthesis of the pigments of life: formation of the macrocycle. Nature (Lond.). 285:17-21.

13. Battersby, A. R., C. J. R. Fookes, G. Hart, G. W. J. Matcham, W. J. George, and P. S. Pandey. 1983. Biosynthesis of porphyrins and related macrocycles. 21. The interaction of deaminase and its product (hydroxymethylbilane) and the relationship between deaminase and cosynthetase. J. Chem. Soc. Perkin Trans. 1:3041-3047.

14. Anderson, P. M., R. Reddy, K. E. Anderson, and R. J. Desnick. 1981. Characterization of the porphyrinogen deaminase deficiency in acute intermittent porphyria. Immunologic evidence for heterogeneity of the genetic defect. J. Clin. Invest. 68:1-12.

15. Anderson, P. M., and R. J. Desnick. 1982. Porphobilinogen deaminase: Methods and principles of the enzymatic assay. Enzyme (Basel). 28:146-157.
16. Corash, L. M., S. Piomelli, H. C. Chen, C. Seaman, and E. Gross. 1974. Separation of erythrocytes according to age on a simplified density gradient. J. Lab. Clin. Med. 84:147-151.

17. Anderson, P. M., and R. J. Desnick. 1980. Purification and properties of $\delta$-aminolevulinate dehydrase from human erythrocytes. J. Biol. Chem. 254:6924-6930.

18. Russell, C. S., and P. Rockwell. 1980. The effects of sulfhydryl agents on the activity of wheat germ uroporphyrinogen I synthase. FEBS (Fed. Eur. Biochem. Soc.) Lett. 116:199-202.

19. Winter, W. P., D. L. Rucknagel, and S. M. Hanash. 1977. Heat denaturation and degradation of hemoglobin. Excerpta Med. Intl. Cong. Ser. 415:307-309.

20. Castro, O., W. P. Winter, R. J. Dean, C. K. Lee, and D. L. Rucknagel. 1976. Heat stability studies in Hemoglobin Agenogi. Clin. Res. 24:630A. (Abstr.)

21. Winter, W. P., D. L. Rucknagel, and C. F. Whitten. 1977. Hemoglobin Hacettepe; $\beta 127(\mathrm{H} 5)$ Gln $\rightarrow$ Glu: a super-stable mutant. Clin. Res. 25:325A. (Abstr.)

22. Orkin, S., and H. H. Kazazian. 1984. Mutation and polymorphism of the human $\beta$-globin gene and its surrounding DNA. Annu. Rev. Genet. 18:131-171.

23. Sassa, S., G. L. Zalar, and A. Kappas. 1978. Studies in porphyria. VII. Induction of uroporphyrinogen I synthase and expression of the gene defect in acute intermittent porphyria in mitogen-stimulated human lymphocytes. J. Clin. Invest. 61:499-508.

24. Sassa, S., S. Granick, D. R. Bickers, R. D. Levere, and A. Kappas. 1973. Studies of the inheritance of human erythrocyte $\delta$ aminolevulinate dehydratase and uroporphyrinogen synthetase. Enzyme (Basel). 16:359-367.

25. Wetterberg, L., V. Floderus, S. Thunell, L. Iselius, and J. Lindsten. 1983. Genetic regulation on the red cell uroporphyrinogen I synthetase level in families with acute intermittent porphyria. Clin. Genet. 24:403-406.

26. Bonaiti-Pellie, C., L. Phung, and Y. Nordman. 1984. Recurrence risk estimation of acute intermittent porphyria based on analysis of porphobilinogen deaminase activity: A Bayesian approach. Am. J. Med. Genet. 19:755-762.

27. Greenberg, D. B., P. V. Tishler, D. A. Holbrook, and J. A. O'Connor. 1978. Multiple alleles for uroporphyrinogen I synthase, the enzyme that is deficient in intermittent acute porphyria. Clin. Res. 26: 615A. (Abstr.) 